\title{
Single-sideband modulator for frequency domain multiplexing of superconducting qubit readout
}

\author{
Benjamin J. Chapman, ${ }^{1,2, a)}$ Eric I. Rosenthal, ${ }^{1,2}$ Joseph Kerckhoff,, ${ }^{1,2, b)}$ Leila R. Vale, ${ }^{3}$ Gene C. Hilton, ${ }^{3}$ and \\ K. W. Lehnert ${ }^{1,2}$ \\ 1) JILA, National Institute of Standards and Technology and the University of Colorado, Boulder, Colorado 80309, \\ $U S A$ \\ ${ }^{2)}$ Department of Physics, University of Colorado, Boulder, Colorado 80309, USA \\ ${ }^{3)}$ National Institute of Standards and Technology, Boulder, Colorado 80305, USA
}

(Dated: 25 May 2017)

\begin{abstract}
We introduce and experimentally characterize a superconducting single-sideband modulator compatible with cryogenic microwave circuits, and propose its use for frequency domain multiplexing of superconducting qubit readout. The monolithic single-quadrature modulators that comprise the device are formed with purely reactive elements (capacitors and Josephson junction inductors) and require no microwave-frequency control tones. Microwave signals in the 4 to $8 \mathrm{GHz}$ band, with power up to $-85 \mathrm{dBm}$, are converted up or down in frequency by as much as $120 \mathrm{MHz}$. Spurious harmonics in the device can be suppressed by up to $25 \mathrm{~dB}$ for select probe and modulation frequencies.
\end{abstract}

Recent advances have allowed many groups to demonstrate superb control over one or several superconducting qubits. This is seen, for example, in the creation and transmission of Fock and cat state superpositions ${ }^{1,2}$, or the ability to continuously tune both the strength ${ }^{3}$ and axis $^{4,5}$ of a quantum non-demolition ${ }^{6}$ measurement. Efforts to combine multiple superconducting qubits into a (more coherent) logical qubit are progressing in parallel..$^{7,8}$ As algorithms on multiple logical qubits become experimentally realizable, the challenge of scaling readout for many-qubit systems grows in relevance.

Multiplexing, a signal processing technique in which a transmission medium is shared among multiple signals, is a natural way to combat such a scaling challenge. In this letter, we introduce a single-sideband modulator (SSBM) for cryogenic microwave applications and propose its use for frequency domain multiplexing of superconducting qubit readout. We describe its design, theory of operation, and experimental performance.

In a circuit quantum electrodynamics architecture, qubits are readout by measuring the transmission of a cavity that has a resonance frequency that is modified (dressed) by a qubit. ${ }^{9}$ Specifically, the cavity resonance is dressed by an amount that depends on the qubit state. To-date, multi-qubit experiments have scaled-up this readout protocol directly ${ }^{10}$ or with frequency domain multiplexing (FDM) hardwired into the measurement architecture. $7,11,12$ In direct "brute-force" scaling, each qubit channel has a dedicated input and output line. Conversely, qubits in an FDM architecture can share a single input and output line, and their readout cavities are designed in advance to ensure their dressed resonances are separated by multiple cavity linewidths.

\footnotetext{
a) Electronic mail: benjamin.chapman@colorado.edu

b) Current address: HRL Laboratories, LLC, Malibu, CA 90265, USA
}

As the number of readout channels in a multi-qubit measurement rises, direct scaling becomes untenable. Recognition of this fact has spurred new proposals for scalable architectures ${ }^{13,14}$, as well as a slew of devices for in-fridge signal processing, from non-reciprocal elements $^{15-20}$ to mixers ${ }^{21,22}$ and switches. ${ }^{14,23,24}$

The "hard-wired" FDM approach achieves scalability at the cost of flexibility. The spectral allotment for each channel is determined prior to fabrication and, unless the readout resonators are designed to be tunable ${ }^{25}$, remains fixed through the measurement process. This constraint inhibits efficient use of the available bandwidth, as densely packing the spectra allotted for each channel makes nearby channels sensitive to deviations in the design parameters. It also precludes the use of identical qubit-cavities, which may be desirable in quantum simulation applications. Finally, the "hard-wired" architecture limits the degree to which a many-qubit experiment can be reconfigured without fabrication of new devices.

There is thus a need for flexible multiplexed architectures that efficiently utilize the available measurement bandwidth. To this end, we introduce a microwave component for cryogenic analog signal processing, which could enable flexible FDM in a many-qubit measurement. This component is a single-sideband modulator (SSBM), a two-port device that converts incident signals up or down in frequency. Unlike commercial SSBMs which utilize resistive diodes, in our device the nonlinear elements are purely reactive. For such systems, the Manley-Rowe relations permit frequency-conversion without dissipation. ${ }^{26}$ Moreover, and in contrast to other cryogenic microwave frequency converters ${ }^{19,27,28}$, the device a) utilizes no resonant physics, endowing it with $\mathrm{GHz}$ of bandwidth, and b) drives its non-linear elements with $\mathrm{RF}$ signal frequencies no greater than several hundred $\mathrm{MHz}$, obviating the need for high-bandwidth $\mathrm{GHz}$ control lines. Finally, the device can be realized as a monolithic integrated circuit in a $\mathrm{NbAlO}_{x} \mathrm{Nb}$ tri-layer process ${ }^{29,30}$, allowing for high-yield wafer-scale production. 


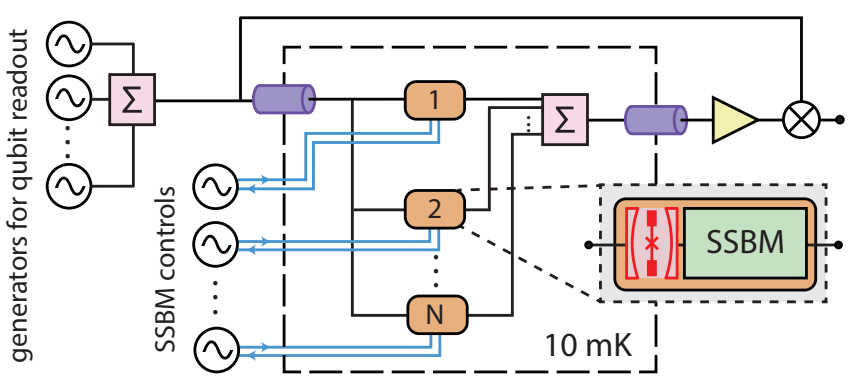

FIG. 1. Conceptual schematic for the proposed frequencydomain-multiplexed readout of $\mathrm{N}$ superconducting qubit/cavity systems (red box) using $\mathrm{N}$ single-sideband modulators (SSBM, green rectangle). The SSBMs frequencyconvert tones exiting the qubit read-out cavities for efficient and dynamically reconfigurable frequency domain multiplexing. As the frequency of the readout tone of each qubit/cavity is converted to a unique portion of the measurement bandwidth, all channels can be readout simultaneously with a single microwave receiver.

A proposed use for the device is shown in Fig. 1. By positioning a SSBM at the output of the readout resonator's strongly coupled port of the qubit/cavity system in each measurement channel, a single microwave line can be used to readout all the channels simultaneously in an FDM scheme. Measurement bandwidth can be dynamically allocated among channels by converting each transmitted tone to its assigned band. This ability to assign spectrum in-situ facilitates efficient use of the available measurement bandwidth, as there is no risk of spectral overlap from uncertainty in fabrication parameters. Furthermore, it allows for the use of FDM even if the dressed cavity frequencies are spectrally irresolvable. Although other multiplexing proposals for time and code-domain schemes ${ }^{14}$ share these benefits, the flexible FDM architecture depicted in Fig. 1 distinguishes itself by requiring no timing coordination between channels.

The SSBM is created from a Hartley-type ${ }^{31}$ in-phasequadrature (IQ) modulator with I and Q ports driven 90 degrees out of phase. This IQ modulator is itself built from a pair of double-balanced modulators (Fig. 2a) realized with inductive bridge circuits (Fig. 2b), which we call Tunable Inductor Bridges (TIBs). ${ }^{14}$ Two pairs of tunable inductors form the bridge, with inductances $l_{1}$ and $l_{2}$. Signals couple differentially to the input port (left and right bridge nodes) and output port (top and bottom bridge nodes). Transmission between these ports is controlled by the imbalance in the bridge $l_{1}-l_{2}$.

Manipulation of that imbalance is accomplished by arranging the two pairs of flux-tunable inductors in a figure-eight geometry. Their coordinated tuning is controlled with an off-chip magnetic coil and an on-chip bias line that bisects the figure eight (Fig. 2c). ${ }^{17}$ Series arrays of Superconducting Quantum Interference Devices (SQUIDs) are used to realize the flux-tunable inductors. Arrays are employed in place of individual SQUIDs to

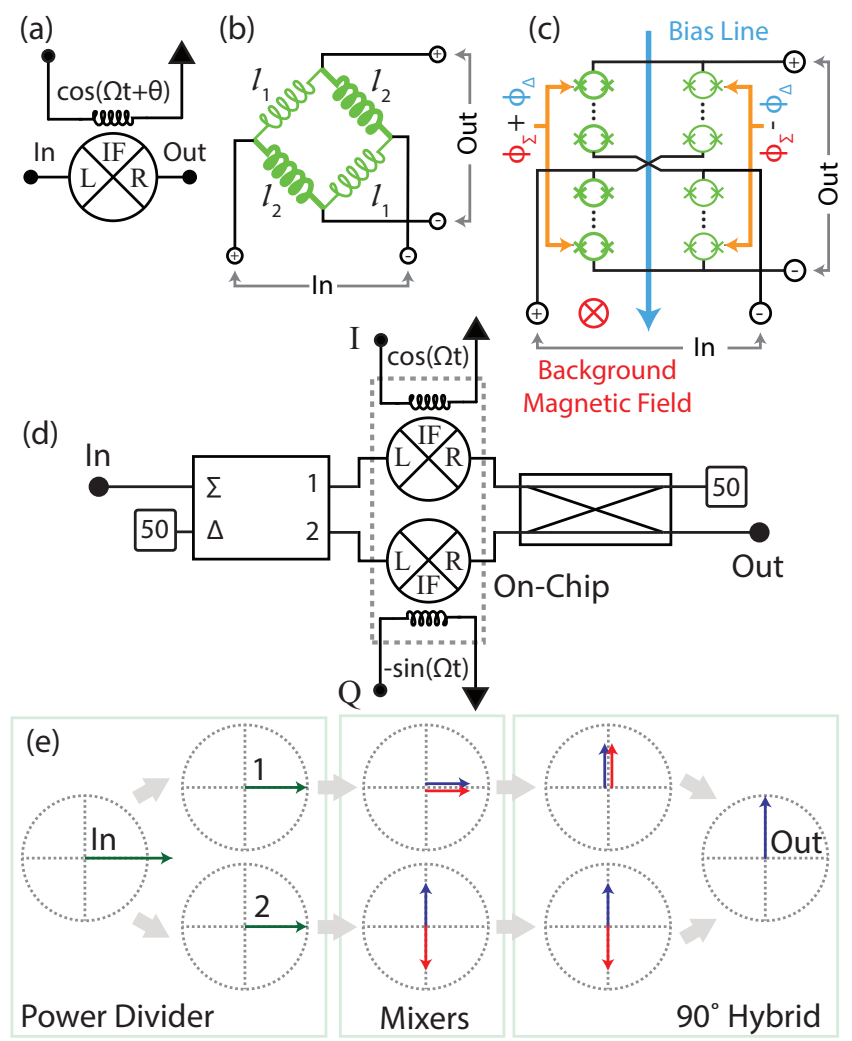

FIG. 2. (a) Double-balanced modulator used to construct the SSBM, realized with a Tunable Inductor Bridge (b) built from two pairs of nominally identical inductors $l_{1}$ and $l_{2}$ arranged opposite one-another. Input and output ports are defined by the left-right and top-bottom nodes of the bridge. Transmission through the bridge scales with the inductor imbalance $l_{1}-l_{2}$. (c) A Tunable Inductor Bridge realized with arrays of SQUIDs. Bridge imbalance is modulated with a static background magnetic flux $\phi_{\Sigma}$ applied with an off-chip magnetic coil and a time-dependent gradiometric flux $\phi_{\Delta}$ applied with an on-chip bias line. (d) Schematic of an SSBM composed of a power divider, two double-balanced modulators with IF ports driven in quadrature, and a 90-degree hybrid coupler. (e) Phasor representation of signals traveling through an idealized SSBM (color indicates relative frequency) during frequency conversion.

dilute the Josephson nonlinearity. ${ }^{17}$

To operate the TIB as a double-balanced modulator, we use the flux-control bias line to sinusoidally modulate the transmission through the bridge at angular frequency $\Omega$. Borrowing language from the field of nonlinearmicrowave elements, we denote the two microwave ports of the TIB as the local oscillator $(\mathrm{L})$ and radio frequency (R) ports of the modulator, and the flux-control line as the intermediate frequency (IF) port. However, as a TIB has no galvanic connections between its IF and L port (or its IF and $\mathrm{R}$ port), we refer to it as a modulator rather than a mixer. Note also that in contrast to a common way of operating mixers by driving their nonlinear elements with a strong signal in their L ports, the IF port 
of the TIB actuates the modulation process and signals at the $\mathrm{L}$ and $\mathrm{R}$ ports can be arbitrarily small.

We create an IQ modulator by dividing the power of an input tone into two double-balanced modulators and then summing their outputs with a 90-degree hybrid coupler (Fig. 2d). In the device reported in this letter, both double-balanced modulators are integrated on a single chip and connected to a commercial power splitter and 90-degree hybrid. Future versions of the SSBM, however, could also integrate the passive components on-chip. ${ }^{24,32}$

Fig. 2e provides a phasor representation of a microwave tone as it propagates through the SSBM. This tone is first split into two arms with equal phase shift, each connected to the L port of a double-balanced modulator. Control currents are applied to the IF ports of both modulators, modulating the signal in each arm into two tones spectrally shifted from the original by $\pm \Omega$ (blue/red sidebands, respectively). The phase of the control current in the lower arm, $\theta$, is advanced $\pi / 2$ radians with respect to that in the upper. Recombination at the 90-degree hybrid causes the blue (red) sidebands in each arm to constructively (destructively) interfere at the device's output. The input tone is thus converted up in frequency by $\Omega$. Generally, the amplitude of the red and blue sidebands scales as $\cos ((\pi / 2 \pm \theta) / 2)$, allowing for selection of the red sideband when $\theta=-\pi / 2$ radians. We denote the IF port in the top arm as the I port of the IQ modulator, and the IF port of the double-balanced modulator in the bottom arm as the $\mathrm{Q}$ port.

This angular dependence of sideband power is depicted in Fig. 3, which shows the transmitted power at various frequencies as a function of the IQ phase difference $\theta$. For this measurement, the SSBM is driven by a microwave tone at $\omega=2 \pi \times 4 \mathrm{GHz}$ through its $\mathrm{L}$ port while we modulate the transmission across the bridges at $\Omega=2 \pi \times$ $3 \mathrm{MHz}$. As this modulation is not purely comprised of a single spectral component at angular frequency $\Omega$, higher harmonics of $\Omega$ at frequencies $\omega \pm n \Omega$, with $n$ an integer greater than 1, are also observed. We monitored the nearest 16 higher harmonics $(2 \leq n \leq 9)$, and found the $n=5$ harmonic to be the largest at this particular choice of $\omega$ and $\Omega$. The plot shows the output of the SSBM at the input frequency (green), along with the first upper (blue) and lower (red) sidebands, and the largest of the higher harmonics ( $n=5$, dashed red and blue). More harmonics and phase sweeps at different IQ modulation frequencies are shown in Fig. S3 of the supplementary material. As $\theta$ is varied between $-\pi$ and $\pi$ we observe that all measured harmonics oscillate with $\theta$ at a rate commensurate with their order.

Two clear operation points are visible at $\theta= \pm \pi / 2$ radians, where the first blue (red) sideband is suppressed in favor of the first red (blue) sideband. The contrast between them exceeds $30 \mathrm{~dB}$ at these points, and the contrast between the desired sideband and the nearest harmonic (the 5th, in this case), which we call the sideband contrast, is approximately $23 \mathrm{~dB}$.

We set the scale of the $\mathrm{y}$-axis in units of $\mathrm{dB}$ relative

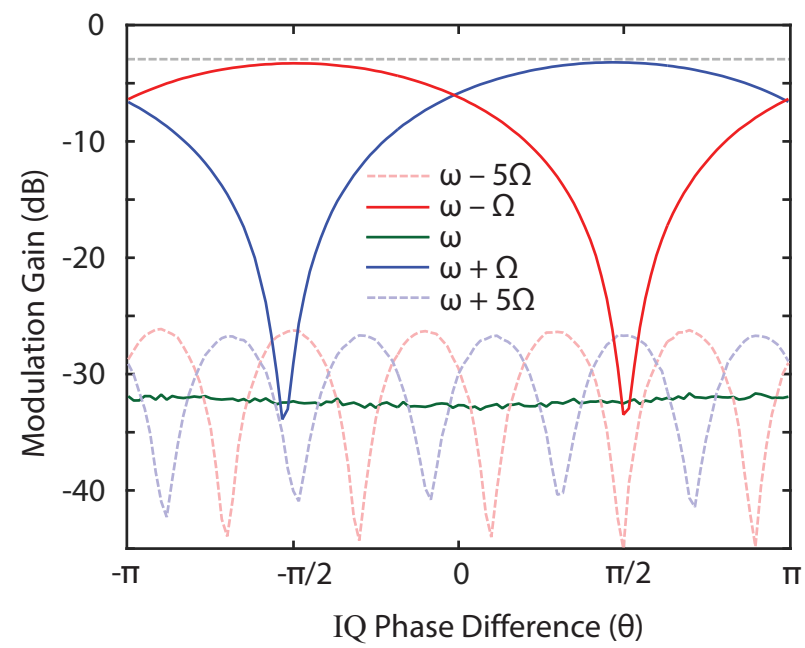

FIG. 3. Modulation gain of circuit shown in Fig. 2d, as a function of IQ phase difference $\theta$, with $\mathrm{L}$ port driven at $\omega=$ $2 \pi \times 4 \mathrm{GHz}$ and transmission modulated at $\Omega=2 \pi \times 3 \mathrm{MHz}$. Note the $3 \mathrm{~dB}$ of power dissipated in the image sideband is not reflected in this measure. The first upper and lower sidebands are shown in solid blue and red, respectively. They exceed the input frequency (green) by up to $30 \mathrm{~dB}$. Power is also detected at higher harmonics of the modulation frequency. For the plotted L and I frequencies, the largest harmonic is the fifth (dashed blue and red, respectively). A gray dashed line at $-3 \mathrm{~dB}$ is a guide to the eye. At the operating points $\theta= \pm \pi / 2$, the power in the first sideband exceeds all other harmonics by more than $20 \mathrm{~dB}$.

to an unmodulated input tone, which we call modulation gain. This is done by statically biasing both TIBs to their state of maximal imbalance. We normalize the sideband power in this way to reveal inefficiencies in the modulation process and separate them from the intrinsic insertion loss of the SSBM's constituent components. In Fig. 3, the modulation gain in the desired sideband never exceeds $-3 \mathrm{~dB}$ (dashed gray line) because the TIB in each arm of the interferometer is biased to modulate transmission sinusoidally. As the device is realized as a distributed network, that means each arm is reflecting half its incident power on average - power which is ultimately dissipated in the power splitter, or directed out the device's input port (return loss).

To characterize the device over a broader range of operation frequencies, sideband contrast for a variety of $\mathrm{L}$ and I frequencies is plotted in Fig. 4a. In the proposed application of the SSBM for FDM of qubit readout, this is an important measure of device performance: when the measurement spectrum is densely filled, spurious sidebands are a source of cross-talk among channels.

In addition to sideband contrast, other important specifications for a SSBM are the modulation gain, L, R, and I frequency limits, instantaneous bandwidth, linearity, and insertion losses. Modulation gain is plotted in Fig. 4b as a function of $\Omega$, for tones at several different input 
(a)

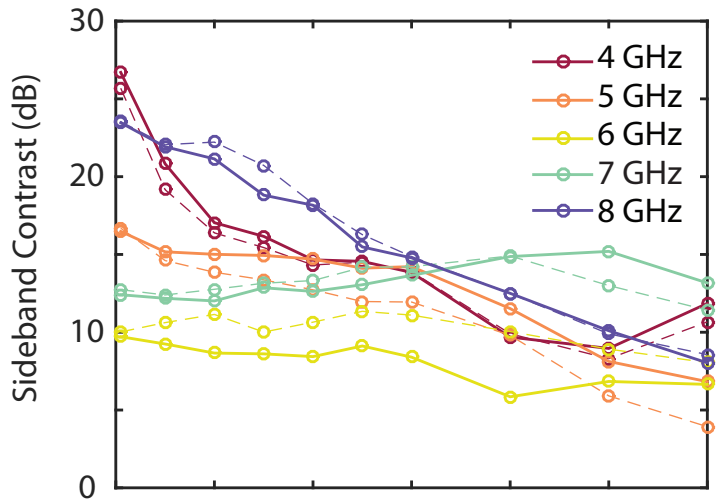

(b)

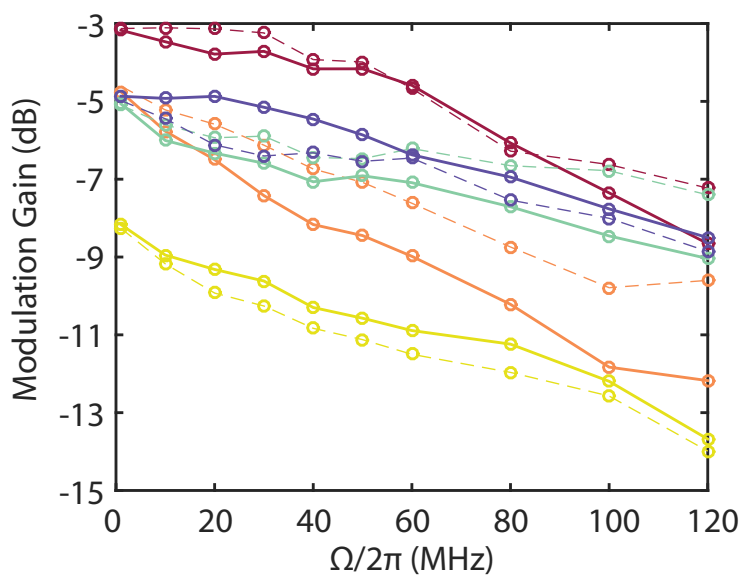

FIG. 4. Sideband contrast (a) and modulation gain (b) of the SSBM, as a function of the modulation frequency $\Omega$. The different traces depict different L frequencies $\omega /(2 \pi)$ (color), for selection of the blue (solid lines) and red (dashed lines) sidebands.

frequencies. Two major trends are visible: first, modulation gain decreases as $\Omega$ increases. This effect is a consequence of the bandwidth requirement of the pulseshaping scheme used to sinusoidally modulate transmission through the TIBs, which begins to exceed our control hardware's bandwidth as $\Omega$ increases. The second trend is a variation in the modulation gain achieved at small modulation frequencies as the probe frequency $\omega$ changes. This is caused by a change in the phase of a TIB's transmission as its magnitude is modulated. This effect is evident in Fig. S2 of the supplementary material, and may be partially alleviated in a future device by removal of a chip-mode at $5 \mathrm{GHz}$.

Figs. $4 \mathrm{a}$ and $\mathrm{b}$ also show the ranges of $\mathrm{L}, \mathrm{R}$, and I frequencies that can be processed by the SSBM. The device converts frequencies from/into the 4 to $8 \mathrm{GHz}$ band, shifting them by as much as $120 \mathrm{MHz}$ to the red or blue. For an FDM application in superconducting qubit readout, the quantity with which to compare this is the coupling strength of a readout cavity's strongly coupled port $\kappa$. Allowing $3 \kappa$ of spectrum per readout channel, and taking a representative value of several $\mathrm{MHz}$ for $\kappa / 2 \pi^{4,7,8,33,34}$, such an I range allows for 10 to 40 (or more ${ }^{35}$ ) independent readout channels per input line.

Conversion of amplitude-modulated tones with the SSBM, and measurements of its linearity, are shown in supplementary Figs. S4 and S5. The instantaneous bandwidth of the device exceeds $5 \mathrm{MHz}$, and its $1 \mathrm{~dB}$ compression point is $-85 \mathrm{dBm}$, providing sufficient bandwidth and power-handling for dispersive qubit readout.

We now discuss the insertion loss of our SSBM. Although the dissipation of the TIBs is less than $0.5 \mathrm{~dB}^{14}$, our SSBM dissipates $3 \mathrm{~dB}$ of the input power (the entire image sideband) in the fourth port of the 90 degree hybrid at the device's output. As noted previously, an additional $3 \mathrm{~dB}$ (or more) of power is dissipated/returned by our distributed network when the TIBs are operated as modulators - this is precisely the modulation gain plotted in Fig. 4b. Finally, the TIBs themselves are not perfectly transmitting over the entire range of operating frequencies. To quantify this, unnormalized transmission in the $4-8 \mathrm{GHz}$ band is plotted in Fig. S2 of the supplementary material, with independent measurements on both TIBs. Although this transmission approaches unity at some frequencies, it is degraded elsewhere by the presence of a chip mode around $5 \mathrm{GHz}$ and impedance mismatches outside the $5-7 \mathrm{GHz}$ band.

A future integrated design for the SSBM could improve on these specifications: removal of the chip mode and matching the circuit impedance across the $4-8 \mathrm{GHz}$ band are well-posed problems in microwave engineering. An on-chip implementation would improve sideband contrast by suppressing path length differences in the network below a wavelength and shrink device footprint by two orders of magnitude. An additional $3 \mathrm{~dB}$ of conversion gain can be recovered by using the quadrature hybrid as the power divider on the input and employing a one-to-one transformer to combine the outputs of the two TIBs - this eliminates the power dissipated in the image sideband. A next-generation device could thus operate over a several $\mathrm{GHz}$ frequency range with insertion loss approaching $-3 \mathrm{~dB}$. Further considerations for a future device are provided in the supplementary material.

A single-sideband modulator constructed from the repeated instancing of broadband modulation elements like TIBs is thus an appealing, general purpose way to engineer frequency conversion. The $\mathrm{L}$ and $\mathrm{R}$ bandwidths allow for conversion of tones in the $4-8 \mathrm{GHz}$ band, and the device's $1 \mathrm{~dB}$ compression point at $-85 \mathrm{dBm}$ far exceeds the power typically ${ }^{36}$ used for dispersive readout. In addition, low return, insertion, and modulation losses are achievable in future design iterations. Finally, the SSBM's nonlinear elements require no $\mathrm{GHz}$ frequency control lines, and are actuated solely with radio frequency signals (several hundred $\mathrm{MHz}$ or less). It is therefore suitable for construction of the scalable and flexible multiplexed architectures needed in future many-qubit experiments with superconducting circuits.

See supplementary material for details on the exper- 
imental setup, bias-waveform pulse shaping, additional measurements, and future design considerations.

Acknowledgment This work is supported by the ARO under contract W911NF-14-1-0079 and the National Science Foundation under Grant Number 1125844. The authors thank Bradley A. Moores and Andrew P. Higginbotham for helpful discussions.

${ }^{1}$ C. Wang, Y. Y. Gao, P. Reinhold, R. Heeres, N. Ofek, K. Chou, C. Axline, M. Reagor, J. Blumoff, K. Sliwa, et al., Science 352, 1087 (2016).

${ }^{2}$ W. Pfaff, C. J. Axline, L. D. Burkhart, U. Vool, P. Reinhold, L. Frunzio, L. Jiang, M. H. Devoret, and R. J. Schoelkopf, arXiv preprint arXiv:1612.05238 (2016).

${ }^{3}$ M. Hatridge, S. Shankar, M. Mirrahimi, F. Schackert, K. Geerlings, T. Brecht, K. M. Sliwa, B. Abdo, L. Frunzio, S. M. Girvin, et al., Science 339, 178 (2013).

${ }^{4}$ S. Hacohen-Gourgy, L. S. Martin, E. Flurin, V. V. Ramasesh,

K. B. Whaley, and I. Siddiqi, Nature 538, 491 (2016).

${ }^{5}$ U. Vool, S. Shankar, S. Mundhada, N. Ofek, A. Narla, K. Sliwa, E. Zalys-Geller, Y. Liu, L. Frunzio, R. Schoelkopf, et al., Physical Review Letters 117, 133601 (2016).

${ }^{6}$ A. Wallraff, D. I. Schuster, A. Blais, L. Frunzio, R.-S. Huang, J. Majer, S. Kumar, S. M. Girvin, and R. J. Schoelkopf, Nature 431, 162 (2004).

${ }^{7}$ J. Kelly, R. Barends, A. G. Fowler, A. Megrant, E. Jeffrey, T. C. White, D. Sank, J. Y. Mutus, B. Campbell, Y. Chen, et al., Nature 519, 66 (2015).

${ }^{8}$ N. Ofek, A. Petrenko, R. Heeres, P. Reinhold, Z. Leghtas, B. Vlastakis, Y. Liu, L. Frunzio, S. Girvin, L. Jiang, et al., Nature (2016).

${ }^{9}$ A. Blais, R.-S. Huang, A. Wallraff, S. M. Girvin, and R. J. Schoelkopf, Physical Review A 69, 062320 (2004).

${ }^{10}$ M. Takita, A. D. Córcoles, E. Magesan, B. Abdo, M. Brink, A. Cross, J. M. Chow, and J. M. Gambetta, Physical Review Letters 117, 210505 (2016).

${ }^{11}$ Y. Chen, D. Sank, P. O'Malley, T. White, R. Barends, B. Chiaro, J. Kelly, E. Lucero, M. Mariantoni, A. Megrant, C. Neill, A. Vainsencher, J. Wenner, Y. Yin, A. N. Cleland, and J. M. Martinis, Applied Physics Letters 101, 182601 (2012), http://dx.doi.org/10.1063/1.4764940.

${ }^{12}$ M. Jerger, S. Poletto, P. Macha, U. Hübner, E. Ilichev, and A. V. Ustinov, Applied Physics Letters 101, 042604 (2012).

${ }^{13}$ T. Brecht, W. Pfaff, C. Wang, Y. Chu, L. Frunzio, M. H. Devoret, and R. J. Schoelkopf, arXiv preprint arXiv:1509.01127 (2015).

${ }^{14}$ B. J. Chapman, B. A. Moores, E. I. Rosenthal, J. Kerckhoff, and K. W. Lehnert, Applied Physics Letters 108, 222602 (2016).

${ }^{15}$ L. Ranzani and J. Aumentado, New Journal of Physics 17, 023024 (2015).

${ }^{16}$ A. Metelmann and A. Clerk, Physical Review X 5, 021025 (2015).

${ }^{17}$ J. Kerckhoff, K. Lalumière, B. J. Chapman, A. Blais, and K. W. Lehnert, Phys. Rev. Applied 4, 034002 (2015).

${ }^{18}$ K. Sliwa, M. Hatridge, A. Narla, S. Shankar, L. Frunzio, R. J. Schoelkopf, and M. H. Devoret, Physical Review X 5, 041020 (2015).

${ }^{19}$ F. Lecocq, L. Ranzani, G. A. Peterson, K. Cicak, R. W. Simmonds, J. D. Teufel, and J. Aumentado, Phys. Rev. Applied 7, 024028 (2017).

${ }^{20}$ B. Abdo, M. Brink, and J. M. Chow, arXiv preprint arXiv:1702.01149 (2017).

${ }^{21}$ N. Bergeal, R. Vijay, V. E. Manucharyan, I. Siddiqi, R. J. Schoelkopf, S. M. Girvin, and M. H. Devoret, Nature Physics 6, 296 (2010).

${ }^{22}$ O. Naaman, J. A. Strong, D. G. Ferguson, J. Egan, N. Bailey, and R. T. Hinkey, Journal of Applied Physics 121, 073904 (2017).

${ }^{23}$ O. Naaman, M. Abutaleb, C. Kirby, and M. Rennie, Applied Physics Letters 108, 112601 (2016).

${ }^{24}$ M. Pechal, J.-C. Besse, M. Mondal, M. Oppliger, S. Gasparinetti, and A. Wallraff, Phys. Rev. Applied 6, 024009 (2016).
${ }^{25}$ J. D. Whittaker, L. J. Swenson, M. H. Volkmann, P. Spear, F. Altomare, A. J. Berkley, B. Bumble, P. Bunyk, P. K. Day, B. H. Eom, R. Harris, J. P. Hilton, E. Hoskinson, M. W. Johnson, A. Kleinsasser, A. Ladizinsky, T. Lanting, T. Oh, I. Perminov, E. Tolkacheva, and J. Yao, Journal of Applied Physics 119, 014506 (2016).

${ }^{26}$ J. Manley and H. Rowe, Proceedings of the IRE 44, 904 (1956). ${ }^{27}$ N. Roch, E. Flurin, F. Nguyen, P. Morfin, P. Campagne-Ibarcq, M. H. Devoret, and B. Huard, Physical review letters 108, 147701 (2012).

${ }^{28}$ B. Abdo, K. Sliwa, F. Schackert, N. Bergeal, M. Hatridge, L. Frunzio, A. D. Stone, and M. Devoret, Physical review letters 110, 173902 (2013).

${ }^{29}$ J. E. Sauvageau, C. J. Burroughs, P. A. A. Booi, M. W. Cromar, R. P. Benz, and J. A. Koch, Applied Superconductivity, IEEE Transactions on 5, 2303 (1995).

${ }^{30}$ J. A. B. Mates, G. C. Hilton, K. D. Irwin, L. R. Vale, and K. W. Lehnert, Applied Physics Letters 92, 023514 (2008).

${ }^{31}$ B. Razavi, RF microelectronics, Vol. 2 (Prentice Hall New Jersey, 1998).

${ }^{32}$ H. Ku, F. Mallet, L. Vale, K. Irwin, S. Russek, G. Hilton, and K. Lehnert, IEEE Transactions on Applied Superconductivity 21, 452 (2011).

${ }^{33}$ W. F. Kindel, M. D. Schroer, and K. W. Lehnert, Phys. Rev. A 93, 033817 (2016).

${ }^{34}$ A. Narla, S. Shankar, M. Hatridge, Z. Leghtas, K. Sliwa, E. ZalysGeller, S. Mundhada, W. Pfaff, L. Frunzio, R. J. Schoelkopf, and M. H. Devoret, Physical Review X 6, 031036 (2016).

35 More readout channels are possible if the cavities in each channel have distinct dressed frequencies or lower cavity coupling rates.

${ }^{36}$ D. Riste, M. Dukalski, C. A. Watson, G. de Lange, M. J. Tiggelman, Y. M. Blanter, K. W. Lehnert, R. N. Schouten, and L. DiCarlo, Nature 502, 350 (2013). 


\section{Supplementary Material for "Single-sideband modulator for frequency domain multiplexing of superconducting qubit readout"}

\section{EXPERIMENTAL SETUP FOR MEASUREMENT OF A SINGLE-SIDEBAND MODULATOR}

A schematic of the experimental setup is shown in Fig. S1. A microwave generator creates a tone that is routed into a ${ }^{3} \mathrm{He}$ cryostat and attenuated. The tone impinges on the sum port of a 180 degree hybrid coupler (Krytar 4040124), which divides the power into two coherent components. The signals then travel through the two TIBs, and are summed together with a 90 degree hybrid (Fairview Microwave SH7219). Finally, the tone propagates through the microwave receiver and is readout in a spectrum analyzer or vector network analyzer.

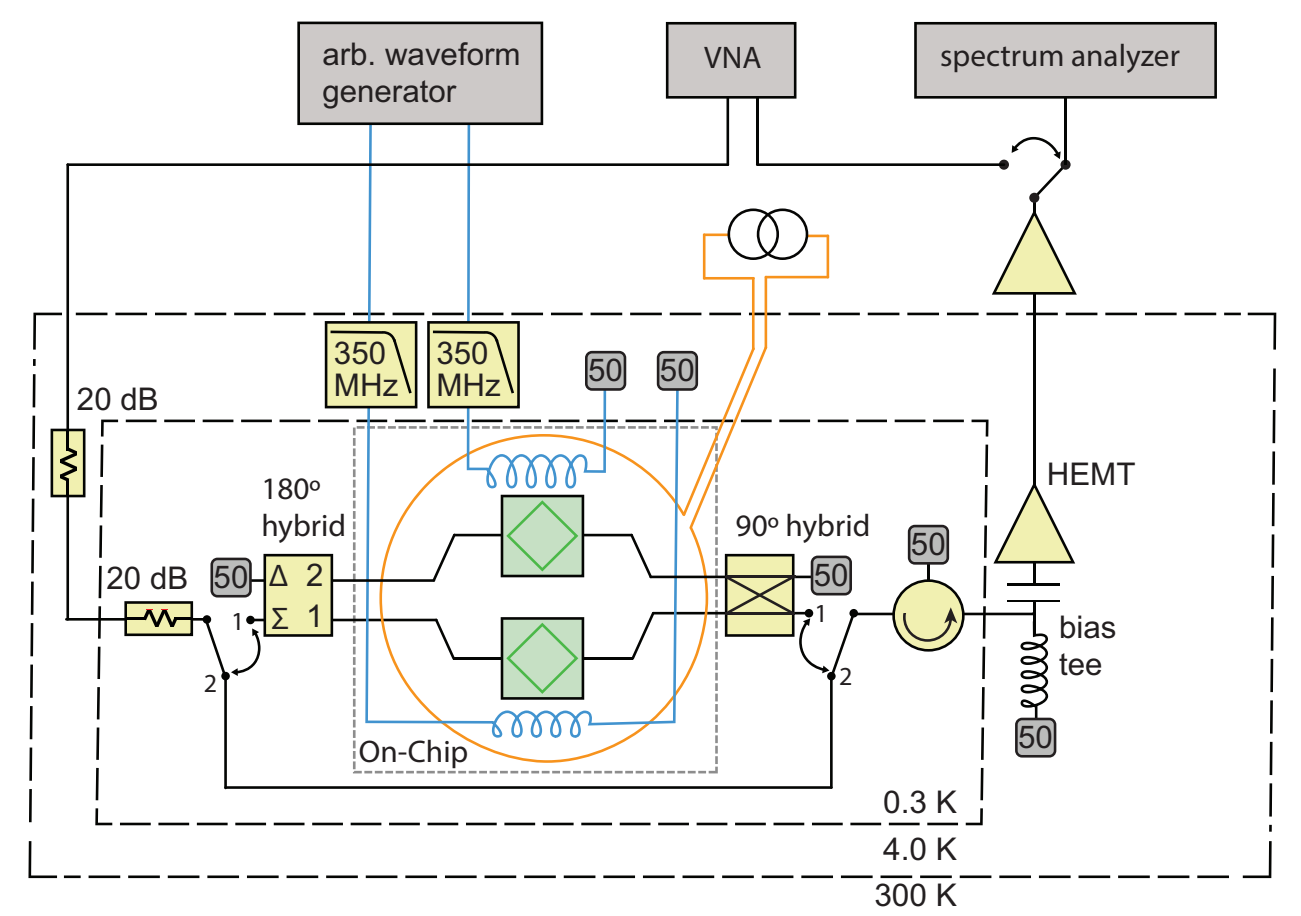

FIG. S1. Wiring diagram for measurements of the single-sideband modulator. TIBs are represented by green diamonds. The orange circle represents an off-chip magnetic coil, and blue inductor symbols represent on-chip gradiometric flux control lines.

To control the transmission of the TIBs, three control currents are used. First, an off-chip magnetic coil (orange circle) creates a uniform magnetic flux $\phi_{\Sigma}$ across the entire chip $(|H| \approx 300 \mathrm{mOe})$. The second and third control currents run in a pair of on-chip bias lines (blue inductors) that create gradiometric fluxes for the two TIBs. To operate a TIB as a double-balanced modulator, we fix the current in the off-chip magnetic coil and dynamically modulate the current $I$ in its on-chip bias line. This changes the imbalance of the TIB, and thus its transmission $T$.

\section{PULSE SHAPING CONTROL CURRENT WAVEFORMS TO OPTIMIZE MODULATOR PERFORMANCE}

Judicious choice of this control current $I$ can substantially improve modulator performance. To calibrate this, we first use a network analyzer to measure the transmission through each TIB as a function of an applied DC current in its on-chip bias line. The other TIB is unbiased during this measurement (its inductor bridge is balanced and incident signals will be reflected) allowing for independent calibration of both TIBs.

Fig. S2a shows the squared amplitude (top row) and phase (bottom row) of signals transmitted through each TIB, as a function of the probe frequency and a gradiometric flux $\phi_{\Delta}$ that is proportional to the control current. In the squared amplitude plots, one observes low transmission when $\phi_{\Delta} / \phi_{0}$ is small, and maximal transmitted power when $\phi_{\Delta} / \phi_{0}=1 / 5$. Here $\phi_{0}=\hbar / 2 e$ is the reduced flux quantum, with $\hbar$ Planck's constant over $2 \pi$ and $e$ the charge of an 
electron. The uniform flux is fixed at $\phi_{\Sigma}=3 \phi_{0} / 10$. Because half the signal is dissipated in the 50 Ohm termination on the 90 degree hybrid, and another half is reflected by the un-probed arm of the network, the maximum possible transmission in this configuration is $-6 \mathrm{~dB}$. From the plots of phase in the bottom row, one can see that inverting the polarity of the bias current causes a $\pi$ phase shift in the transmission.
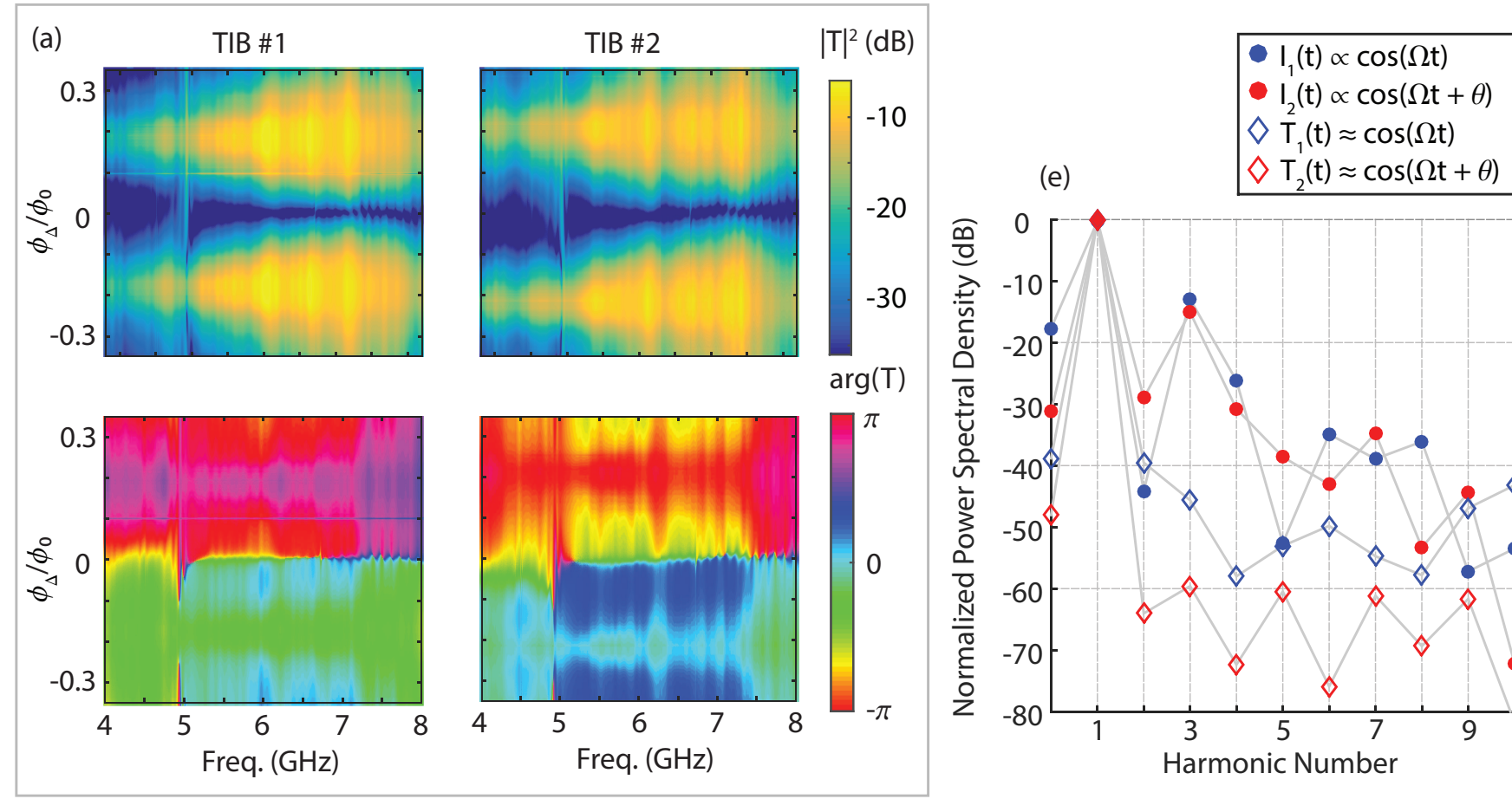

(b)

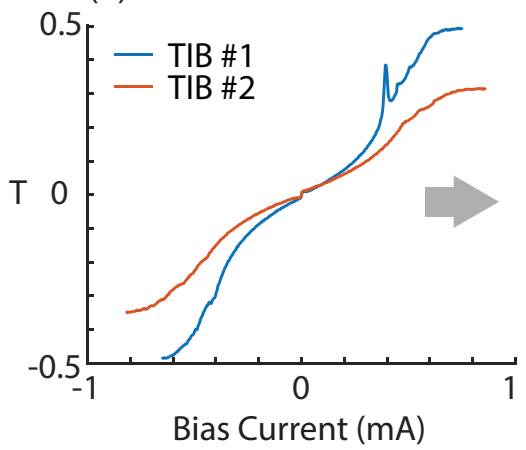

(c)

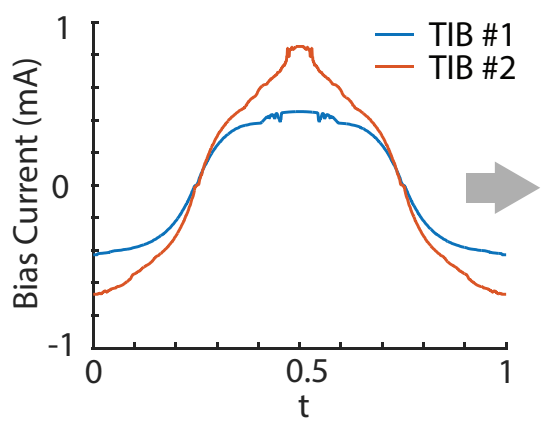

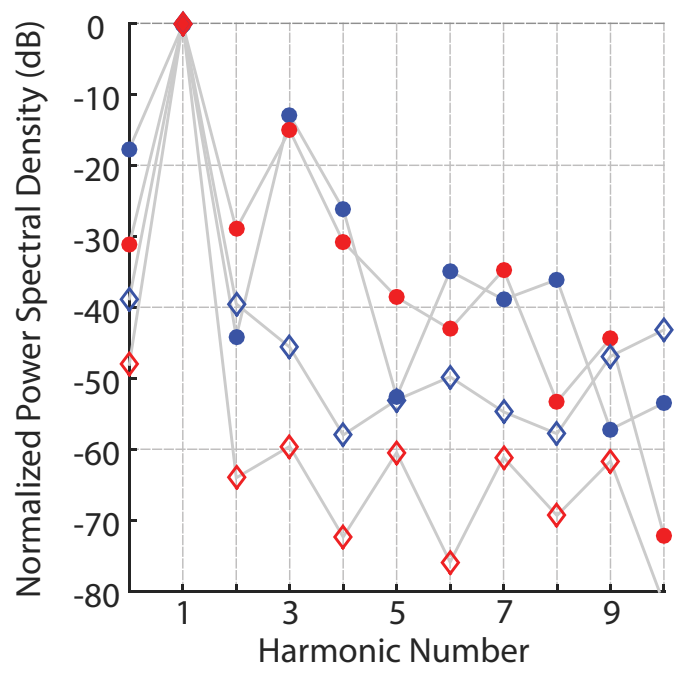

(d)

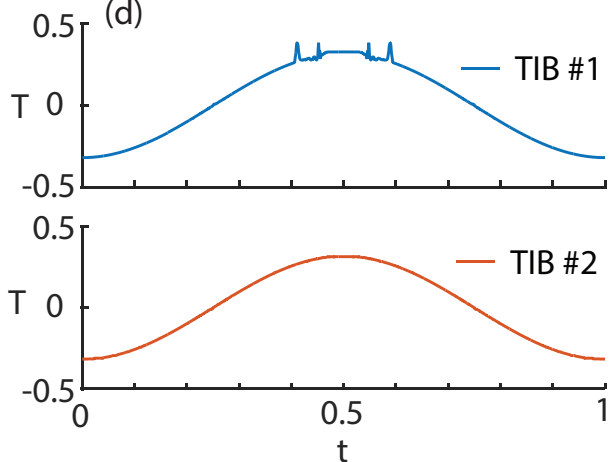

FIG. S2. (a) Amplitude and phase of transmission measurements on each TIB, while the other TIB is set to reflect incident signals (zero bias current). The maximum of the transmission color scale is $-6 \mathrm{~dB}$. The phase of transmission shifts by approximately 180 degrees as the bias current changes polarity. (b) Linecuts of transmission vs bias current $T(I)$, with probe frequency fixed at $6 \mathrm{GHz}$. When bias current is swept sinusoidally, transmission changes in a non-sinusoidal manner. (c) Applying a bias current $I(t)=T^{-1}(\cos (\Omega t))$ produces approximately sinusoidal modulation of transmission, as seen in $(\mathrm{d})$. For TIB \#1, the map $T(I)$ is not invertible, which causes some distortion (higher harmonics of $\Omega$ ) in the transmitted spectrum. (e) Power spectral density of transmission when current is modulated sinusoidally (filled circles), and when the current is chosen to produce sinusoidal transmission (unfilled diamonds). Spectra are normalized to the total power in each sideband. Pulse shaping reduces the weight of unwanted higher harmonics by approximately $20 \mathrm{~dB}$. Data for TIB\#1 (TIB\#2) are shown in blue (orange).

Linecuts of the real part of the transmission at $6 \mathrm{GHz}$ are shown in Fig. S2b. These are maps $T(I)$ between the applied control current and the transmission through the bridges. If these maps were linear, a sinusoidal control current would induce a sinusoidal modulation of the transmission through the TIB. Transmission, however, is a nonlinear function of the control current. ${ }^{\mathrm{S} 1}$ To ensure sinusoidal modulation by the TIBs at the desired angular frequency $\Omega$, we 
use an arbitrary waveform generator to apply the control current $I(t)=T^{-1}(\cos (\Omega t))$ (Fig. S2c). Here $T^{-1}$ denotes the inverse of the map $T(I)$. The time-dependence of the transmission, $T(t)$, is then ensured by function composition to be the desired value of $\cos (\Omega t)$ (Fig. S2d). When $T(I)$ is not a one-to-one mapping (Fig. S2b, blue curve) this procedure only produces an approximation of sinusoidal modulation, as seen in Fig. S2d, top panel. (As the optimal bias current waveform $I(t)$ must be sampled discretely before loading onto the arbitrary waveform generator and is bandwidth limited by that arbitrary waveform generator, in practice $T(t)$ is a smoother function than that shown in Fig. S2d.) The overall effect of this procedure can be seen in Fig. S2e, which plots the power spectral density of $T(t)$ for both TIBs, when the bias currents are sinusoidal (circles) and when the bias currents are chosen to produce sinusoidal transmission (diamonds). The latter method reduces the spectral weight of higher harmonics by about 20 dB.

\section{IF FREQUENCY LIMITATIONS}

Harmonics in the spectrum of $T(t)$ are imprinted directly onto the spectrum of signals transmitted through the SSBM. Fig. S3 shows the nearest 10 harmonics around as the IQ phase difference is swept. The LO port of the device is driven by a microwave tone at $\omega=2 \pi \times 4 \mathrm{GHz}$ and the I and $\mathrm{Q}$ ports are driven by angular frequencies of $\Omega=2 \pi \times 3 \mathrm{MHz}$ (Fig. S3a), $\Omega=2 \pi \times 20 \mathrm{MHz}$ (Fig. S3b), and $\Omega=2 \pi \times 60 \mathrm{MHz}$ (Fig. S3c).

(a) $\Omega / 2 \pi=3 \mathrm{MHz}$

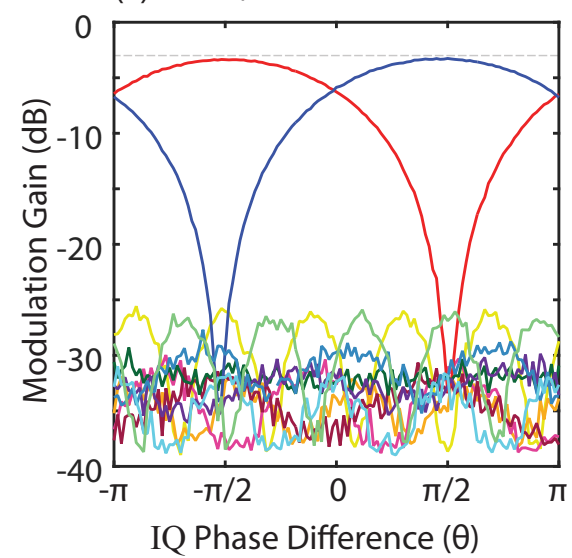

(b) $\Omega / 2 \pi=20 \mathrm{MHz}$

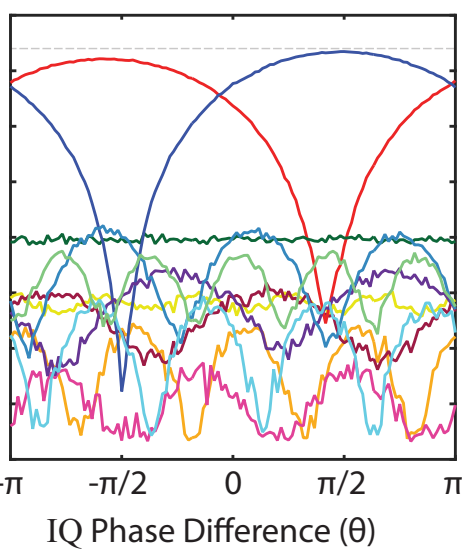

(c) $\quad \Omega / 2 \pi=60 \mathrm{MHz}$

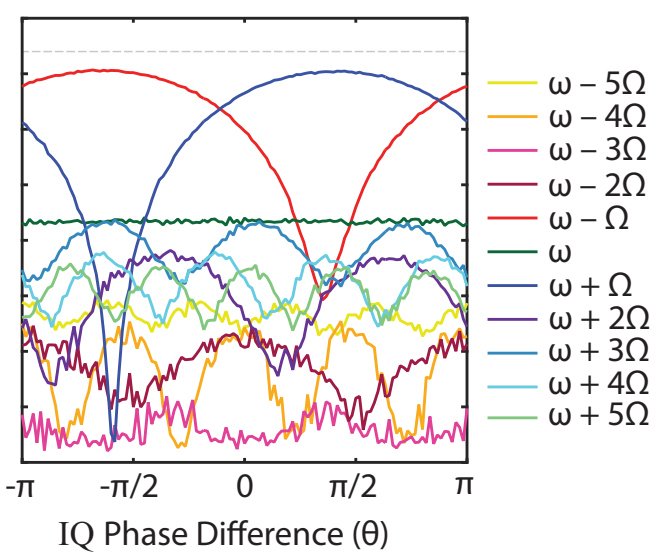

FIG. S3. Sideband power as a function of IQ phase difference for modulation rates of $\Omega / 2 \pi=3 \mathrm{MHz}$ (a), $20 \mathrm{MHz}$ (b), and 60 $\mathrm{MHz}(\mathrm{c})$, with $\omega / 2 \pi=4 \mathrm{GHz}$. A gray dashed line at $-3 \mathrm{~dB}$ shows the expected upper-bound on transmitted power.

As $\Omega$ is increased and signals are converted farther from the input frequency, the output at both the input frequency and at spurious harmonics increase in magnitude. This is a consequence of the finite bandwidth of our arbitrary waveform generator (Tektronix AWG5014c), which samples at $1.2 \mathrm{Gs} / \mathrm{s}$ and has a bandwidth of $180 \mathrm{MHz}$. (For input powers much less than the $1 \mathrm{~dB}$ compression point, the size of spurious harmonics does not depend on the power of the input signal.) As the IF modulation rate $\Omega$ grows, the number of points available to represent the control current pulse $I(t)$ decreases, and the bandwidth needed to capture fine corrections to $I(t)$ increases. This diminishes the ability of the pulse-shaping routine to ensure approximately sinusoidal transmission. For instance, as $\Omega /(2 \pi)$ changes from 3 to $60 \mathrm{MHz}$, the image rejection goes from $30 \mathrm{~dB}$ to $20 \mathrm{~dB}$, and the sideband contrast goes from $25 \mathrm{~dB}$ to 13 dB.

\section{FREQUENCY CONVERSION OF AMPLITUDE-MODULATED MICROWAVES}

The previous sections shows frequency conversion of pure microwave tones with an SSBM. In the envisaged application, however, the SSBM must convert wavepackets with more complex spectral composition. A realistic example of such a signal is a microwave tone that is phase modulated in time, at a rate set by the bandwidth of the qubit readout cavity. Such a signal simulates the dataflow from a qubit-cavity system that is being continuously monitored, say, for the detection of quantum jumps: as the qubit state stochastically jumps between ground and excited, the phase of the transmitted microwave tone concomitantly acquires a phase shift of 0 or $\pi$ radians. The phase modulation of the 
transmitted signal creates a wavepacket with a richer frequency content than the orginal tone, and extraction of the information in the phase modulation requires single-sideband modulation of the entire wavepacket.

To simulate this process, we use the SSBM to frequency-convert a microwave tone that is amplitude modulated with a square wave at frequency $\nu$. (For this demonstration, we use amplitude modulation in place of phase modulation to allow detection with a spectrum analyzer.) We measure the modulation of the signal's amplitude in time (Fig. S4a-c), and the spectrum of the converted signal (Fig. S4d-f). Amplitude modulations of $\nu=100 \mathrm{KHz}, 1 \mathrm{MHz}$, and $5 \mathrm{MHz}$ are shown in the top, middle, and bottom rows of the figure. For each modulation, the four traces show frequency conversion by $0,3,20$, or $60 \mathrm{MHz}$, as evidenced by the shifted spectra in the figure's right column.
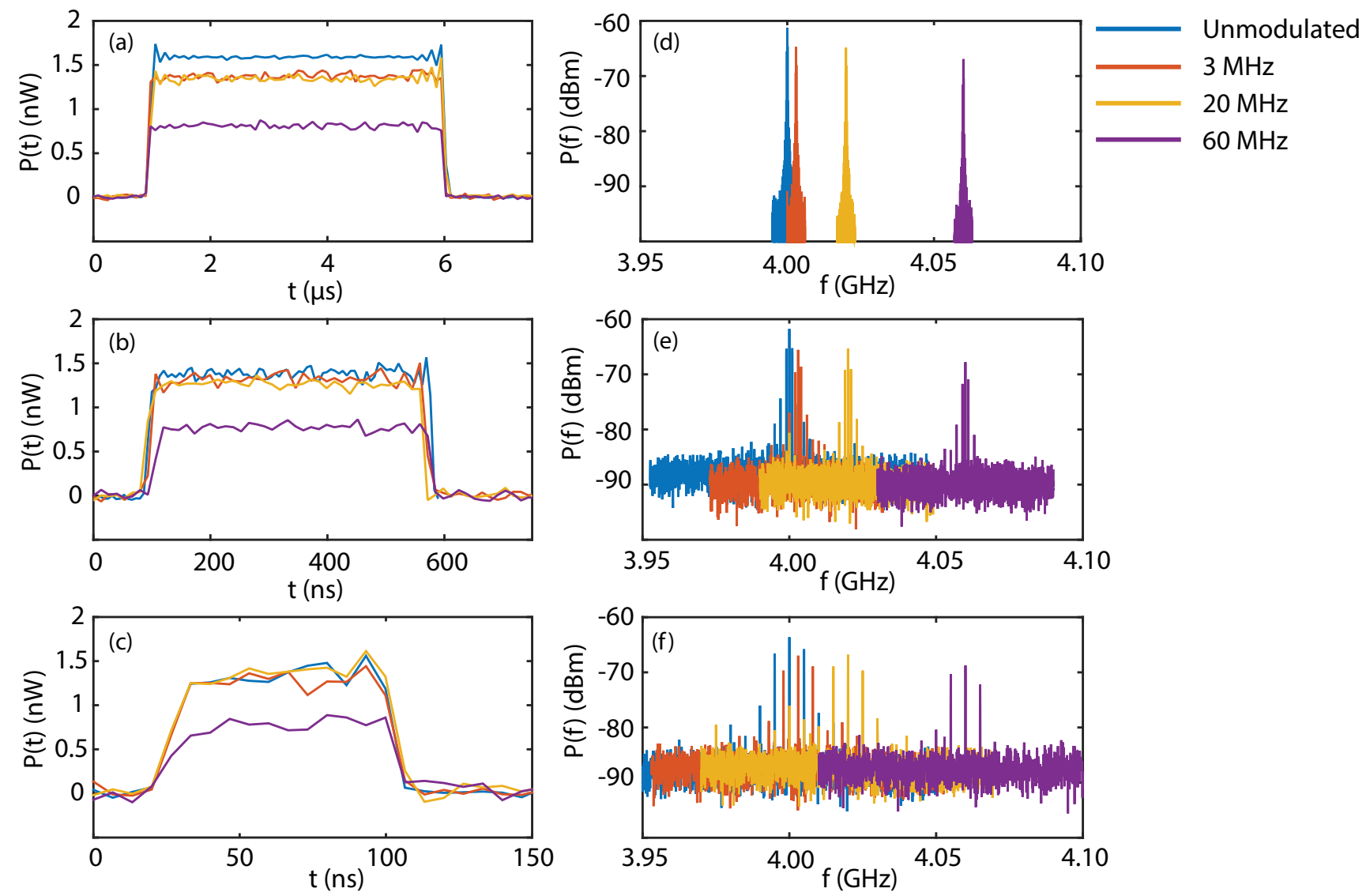

FIG. S4. Amplitude modulation of an $\omega=2 \pi \times 4 \mathrm{GHz}$ tone after frequency conversion by the SSBM. Transmitted power versus time (a-c) and power spectral density (d-f) are shown for modulation rates of $100 \mathrm{KHz}$ (top row), $1 \mathrm{MHz}$ (middle row), and 5 $\mathrm{MHz}$ (bottom row). At each of the three modulation rates, we use the SSBM to convert the frequency of an input tone by 0 , 3, 20, and $60 \mathrm{MHz}$. These are depicted by the four traces in each panel. For visual clarity, the amplitude of the unconverted signals (blue traces) in (a-c) are scaled by a factor of $1 / 2$.

From Fig. S4, one can see that the instantaneous bandwidth of the SSBM exceeds $5 \mathrm{MHz}$. At higher frequencies, for example $5 \mathrm{MHz}$, distortion of the square-wave begins to become apparent. We believe the instantaneous bandwidth of the device is currently limited by the relaxation of eddy currents in the chip ground plane. This relaxation time can be substantially reduced in future devices.

\section{LINEARITY}

To assess the power-handling of the SSBM we sweep the on-chip power during operation at $\Omega / 2 \pi=3 \mathrm{MHz}$, $\omega / 2 \pi=4 \mathrm{GHz}$, and $\theta=90$ degrees (up-conversion), and measure the power transmitted at various harmonics of the modulation rate. Fig. S5 shows the results. We measure the power in the nearest 18 harmonics $(n \leq 9)$ to the input frequency. For visual clarity, only the zeroth and the four lowest odd harmonics are plotted, though the other harmonics exhibit similar behavior. 


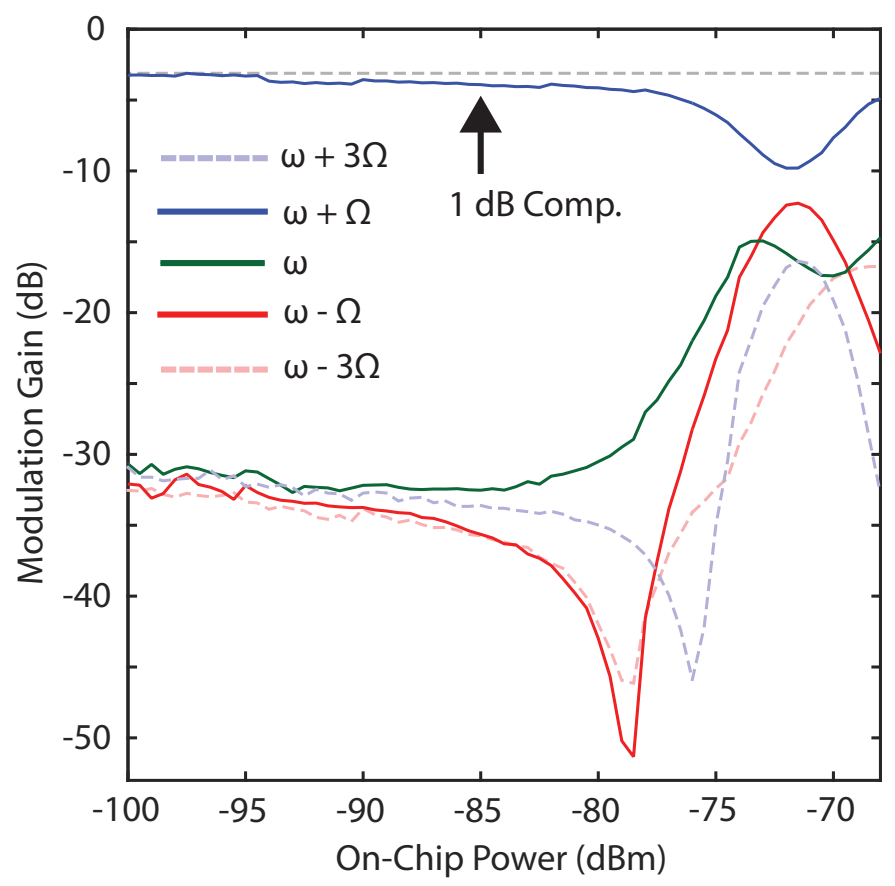

FIG. S5. Selected sideband power as a function of on-chip power, with $\omega / 2 \pi=4 \mathrm{GHz}, \Omega / 2 \pi=3 \mathrm{MHz}$, and $\theta=90$ degrees. 1 $\mathrm{dB}$ compression occurs at $-85 \mathrm{dBm}$. Dashed line at $-3 \mathrm{~dB}$ is a guide to the eye.

When the incident power is below $-95 \mathrm{dBm}$, the output power at the input frequency and in all the sidebands scales linearly. As the input power increases, $1 \mathrm{~dB}$ compression in the first blue sideband occurs at $-85 \mathrm{dBm}$. This value is consistent with previous results, which found the $1 \mathrm{~dB}$ compression point of a single TIB to be $-88 \mathrm{dBm}^{\mathrm{S} 1}$. (As the TIBs in our SSBM need only process one-half the power incident on the device's input, the SSBM's compression point is $3 \mathrm{~dB}$ below that of a single TIB.) In a dispersive readout scheme the power of a readout tone depends on the dressed cavity bandwidth, but most tones fall between -130 and $-120 \mathrm{dBm}^{\mathrm{S} 2}$. The SSBM's $1 \mathrm{~dB}$ compression point at $-85 \mathrm{dBm}$ thus provides ample power handling for this application.

\section{CONSIDERATIONS FOR A FUTURE, ON-CHIP, INTEGRATED DESIGN}

As the SSBM is created with two instances of a TIB, design improvements for the single-sideband modulator may be partitioned into two categories: changes of the TIB, and changes of the composite device (the two TIBs, hybrid couplers, and internal microwave connections). We address these in turn.

As mentioned in the main text, immediate improvements on the TIB may be realized by eliminating the chip-mode around $5 \mathrm{GHz}$ (apparent in Fig. S2a), and by improving the impedance match. The chip-mode is an LC resonance between the inductance of the balun coils and a parasitic capacitance to ground, which can be tuned out of the operation band by manipulating the width of the balun microstrips (their length is constrained to be a quarter wavelength at the operation frequency).

In the current generation of devices, impedance matching a TIB to 50 Ohms is inhibited by the finite tunability of the inductors in the bridge and the impedance of those inductors (about $20 \mathrm{Ohms}$ at $6 \mathrm{GHz}$ ) with respect to 50 Ohms. We overcome this problem by tuning out the residual inductance with capacitors in series with the four nodes of the bridge. Although this is an expedient solution, the resulting match is effective only within several hundred $\mathrm{MHz}$ of the frequency for which the capacitors have the appropriate impedance. A more sophisticated approach, such as tapered lines or matching transformers, could easily expand this bandwidth to several $\mathrm{GHz}$, as the Bode-Fano criterion $^{\mathrm{S} 3, \mathrm{~S} 4}$ is relatively lenient for low $Q$ circuits. More quantitatively, an imbalanced TIB may be modeled as an inductor in series with a $50 \mathrm{Ohm}$ load. The Thevenin equivalent inductance of a TIB with inductors parametrized as $l_{1}$ and $l_{2}$ (as in Fig. $2 \mathrm{~b}$ of the main text) is $2 l_{1} l_{2} /\left(l_{1}+l_{2}\right)$, which for the TIBs in this device amounts to $800 \mathrm{pH}$ when the bridges are maximally imbalanced. The Bode-Fano criterion thus allows for $-20 \mathrm{~dB}$ reflections over an $8 \mathrm{GHz}$ bandwidth. Improved impedance matching may therefore drastically reduce return loss over the 4 to $8 \mathrm{GHz}$ band. 
Better impedance matching can also be achieved directly by improving the tunability (dynamic range) of the SQUID critical currents. While reducing the number of SQUIDs in each array will accomplish this by reducing geometric inductance and enhancing participation ratios, power handling will suffer. Another way to improve tunability is to more uniformly distribute the gradiometric flux across the length of the array, which reduces variation in the critical currents of the SQUIDs that make up the array. In the current layout, SQUIDs near the inside corners of the bias line couple more strongly to it, and are threaded by greater amounts of flux. Laying out the figure-eight of the bridge so that the bias line can bisect it without making turns will alleviate this problem, increase the degree to which the bridge can be imbalanced, and reduce impedance mismatch.

Finally, the instantaneous bandwidth of the TIB may also be improved by elimination of long timescale eddy currents in the chip ground plane. This can be done by altering the resistance (modifying the $L / R$ time) of a normal metal layer placed in the chip ground plane to break supercurrent loops.

In addition to improving the constituent components, a next-generation SSBM can also benefit from complete onchip integration. First, on-chip superconducting hybrids can be extremely low-loss. Second, path length differences in the two arms of the network can be easily constrained to micron length scales, making them smaller than the operation wavelengths by more than four orders of magnitude. (To contrast, in our current device this path length difference is $3 \mathrm{~mm}$ ). Suppressing path length difference far below the operation wavelength allows for simultaneous minimization of the power in the image sideband and maximization of the power in the desired sideband.

A third considerable benefit of moving to a completely monolithic circuit is the recovery of an additional $3 \mathrm{~dB}$ of modulation gain. In the current implementation, an off-chip power splitter divides the input power and delivers it to the two on-chip TIBs. During operation, this distributed network suffers from return loss, as each TIB is mismatched from $50 \mathrm{Ohms}$ during portions of the modulation period (a portion of this reflected power is dissipated in the power divider at the input, and another portion exits the device's input as return loss). It also results in an additional $3 \mathrm{~dB}$ of insertion loss; when signals in the two arms of the interferometer are summed in the 90 degree hybrid, the image (undesired) sideband is dissipated in the terminated port of the hybrid.

To remedy this, we propose using a 90-degree hybrid coupler as the power-divider at the device's input, and combining the signals in the interferometer with a one-to-one transformer. Although this layout does not recover the $3 \mathrm{~dB}$ of return and insertion loss at the device's input, it eliminates the $3 \mathrm{~dB}$ of insertion loss from the image sideband at the output. An SSBM designed in this way could operate with a modulation gain of $-3 \mathrm{~dB}$.

[S1]B. J. Chapman, B. A. Moores, E. I. Rosenthal, J. Kerckhoff, and K. W. Lehnert, Applied Physics Letters 108, 222602 (2016).

[S2]D. Riste, M. Dukalski, C. A. Watson, G. de Lange, M. J. Tiggelman, Y. M. Blanter, K. W. Lehnert, R. N. Schouten, and L. DiCarlo, Nature 502, 350 (2013).

[S3]H. W. Bode et al., Network analysis and feedback amplifier design (van Nostrand, 1945).

[S4]R. M. Fano, Journal of the Franklin Institute 249, 57 (1950). 